\title{
Dinámica de nutrientes en plantaciones forestales de Azadirachta indica (Meliaceae) establecidas para restauración de tierras degradadas en Colombia
}

\author{
Claudia Patricia Flórez-Flórez ${ }^{1}$, Juan Diego León-Peláez ${ }^{1}$, Nelson Walter Osorio-Vega ${ }^{2}$ \& \\ Manuel Fernando Restrepo-Llano ${ }^{1}$ \\ 1. Facultad de Ciencias Agropecuarias, Departamento de Ciencias Forestales, Universidad Nacional de Colombia; Calle \\ 59A 63-20,14-330; claudiapatriciaflorez@gmail.com, jdleon@unal.edu.co, manuelfernando7@gmail.com \\ 2. Facultad de Ciencias, Escuela de Geociencias, Universidad Nacional de Colombia; nwosorio@unal.edu.co
}

\author{
Recibido 28-III-2012. Corregido 10-X-2012. Aceptado 16-X-2012.
}

\begin{abstract}
Nutrient dynamics in forest plantations of Azadirachta indica (Meliaceae) established for restoration of degraded lands in Colombia. Azadirachta indica is a tree species which use is steadily increasing for restoration of tropical and subtropical arid and degraded lands throughout the world. The objective of this research study was to evaluate the potential of these plantations as an active restoration model for the recovery of soils under desertification in arid lands of Colombia. Litter traps and litter-bags were installed in twenty $250 \mathrm{~m}^{2}$ plots. Green leaves and soil samples inside and outside this species plantations were taken, and their elemental concentrations were determined. Litterfall, leaf litter decomposition and foliar nutrient resorption were monitored for one year. The annual contributions of organic material, such as fine litterfall, represented $557.54 \mathrm{~kg} / \mathrm{ha}$, a third of which was $A$. indica leaves. The greatest potential returns of nutrients per foliar litterfall were from $\mathrm{Ca}(4.6 \mathrm{~kg} / \mathrm{ha})$ and $\mathrm{N}(2.4 \mathrm{~kg} / \mathrm{ha})$, and the smallest potential returns came from P $(0.06 \mathrm{~kg} / \mathrm{ha})$. A total of $68 \%$ of the foliar material deposited in litter-bags disappeared after one year. The greatest release of nutrients was that of $\mathrm{K}(100 \%)$, and the least was that of $\mathrm{N}(40 \%)$. P was the most limiting nutrient, with low edaphic availability and high nutrient use efficiency from Vitousek's index $(I E V=3176)$ and foliar nutrient resorption (35\%). Despite these plantations are young, and that they have not had forestry management practices, as an active restoration model, they have revitalized the biogeochemical cycle, positively modifying the edaphic parameters according to the increases in organic material, $\mathrm{P}$ and $\mathrm{K}$ of $72 \%, 31 \%$ and $61 \%$, respectively. Furthermore, they improved the stability of aggregates and the microbe respiration rates. The forest plantation model with exotic species has been opposed by different sectors; however, it has been acknowledged that these projects derive many benefits for the restoration of biodiversity and ecosystemic functions. The conditions of severe land degradation demand the initial use of species, such as $A$. indica, that can adapt quickly and successfully, and progressively reestablish the biogeochemical cycle. Rev. Biol. Trop. 61 (2): 515-529. Epub 2013 June 01.
\end{abstract}

Key words: soil rehabilitation, restoration ecology, biogeochemical cycle, Neem (Azadirachta indica), litterfall, litter decomposition, nutrient return.

La degradación o desertificación en tierras de zonas áridas, semiáridas y subhúmedas secas, representa un problema de importantes repercusiones ambientales, resultado principalmente de las variaciones climáticas y de las actividades humanas (UN 1994, Le Houèrou 1975, Reynolds \& Stafford-Smith 2002). Así, el desmonte y cultivo inadecuado, el pastoreo excesivo y la recolección indiscriminada de plantas leñosas, responden por cerca del $80 \%$ de la degradación de las tierras áridas (Le Houèrou 1976, Anaya 1986).

En Colombia, el reemplazo de la vegetación original de las zonas secas, por pastos para ganado, ha conllevado a la pérdida progresiva del Bosque Seco Tropical, considerado entre los tres ecosistemas más degradados, fragmentados y menos conocidos del país. 
Así, de los bosques secos a subhúmedos persisten en la actualidad, cerca de $1200 \mathrm{~km}^{2}$ de los $80000 \mathrm{~km}^{2}$ originales (MAVDT 2004). Aunque para la recuperación de ecosistemas degradados, los modelos de restauración pasiva basados en procesos de regeneración natural son sencillos y económicos, no siempre son exitosos (Holl 2002, Schrautzer et al. 2007). Alternativamente, los modelos de restauración activos permiten, además de restaurar el hábitat para la biodiversidad, acelerar el restablecimiento de procesos ecológicos como el ciclo de nutrientes y el secuestro de carbono (Celentano et al. 2011).

El modelo más común de restauración activa es la plantación de árboles en altas densidades. Éste ha revelado la utilidad de las plantaciones forestales para la recuperación del suelo y de la diversidad biológica en tierras tropicales degradadas (Garten 2002, Singh et al. 2002), como consecuencia de la reactivación del ciclo biogeoquímico vía producción y descomposición de hojarasca. Estos procesos conducen, entre otros aspectos, al incremento de materia orgánica y de nutrientes en el suelo, a la moderación del pH, al mejoramiento de la estabilidad de agregados, y al incremento de la capacidad de almacenamiento de agua (Chakraborty \& Chakraborty 1989). Las especies vegetales que se incorporen en modelos de restauración y/o productivos, deben contar con una alta capacidad de adaptación a las condiciones extremas de degradación y un eficiente reciclado externo de los nutrientes edáficos a través de procesos del ciclo biogeoquímico (Ferrari \& Wall 2004), hacer altos aportes de materia orgánica al suelo como hojarasca, y mostrar una alta eficiencia en el uso de los nutrientes (Sayer \& Elliot 2005, León et al. 2011).

La utilización de plantas no autóctonas para un proyecto específico de restauración es aceptada por la Sociedad Internacional para la Restauración Ecológica (SER International), dado que se reconoce que estas especies cumplen con roles ecológicos previamente desempeñados por las especies autóctonas, aclarando que la posterior extirpación eventual de la especie debe incluirse en el plan de ejecución de la estrategia de restauración. Azadirachta indica A. Juss (Nim) ha sido ampliamente empleada en procedimientos de restauración, tanto bajo arreglo en plantación monoespecífica como mixta, para la recuperación de suelos degradados en zonas secas (Radwanski \& Wickens 1981). Características como la rápida tasa de descomposición de sus hojas han motivado su consideración en programas de mejoramiento de la fertilidad del suelo en terrenos pedregosos y arenosos con tendencia a la desertificación (Radwanski \&Wickens 1981). Asimismo ha sido utilizada para la rehabilitación de suelos alterados por minería (Singh et al. 2000), para la recuperación de suelos afectados por salinidad (Singh \& Garg 2007), y para la recuperación de zonas degradadas por minería de carbón (Mehrotra 1998).

El objetivo de este estudio fue valorar el potencial de plantaciones de A. indica como modelo de restauración activa, para la recuperación de suelos degradados. Para ello se caracterizaron procesos del ciclo biogeoquímico, relacionados con los flujos de materia orgánica y nutrientes desde el dosel (producción de hojarasca y retorno potencial de nutrientes, descomposición de hojarasca y liberación de nutrientes, reabsorción y eficiencia en el uso de nutrientes) y se valoraron parámetros físicos y químicos de los suelos, con el fin de observar la evolución de las propiedades edáficas en los sitios plantados, tomando como punto de referencia sitios no plantados degradados.

\section{MATERIALES Y MÉTODOS}

Área de estudio: El estudio se realizó en Santa Fe de Antioquia, cuenca del río Cauca, al noroeste de Colombia (632'28' N - 75 $48^{\circ} 6^{\prime}$ " W, 542msnm). Los valores medios anuales de temperatura, brillo solar y evaporación son $26.6^{\circ} \mathrm{C}, 2172 \mathrm{hr}$ y $1594 \mathrm{~mm}$, respectivamente (Estación meteorológica Cotové, 530msnm, $\left.6^{\circ} 33^{\prime} 59^{\prime \prime} \mathrm{N}-75^{\circ} 50^{\prime} 9 " \mathrm{~W}\right)$. El paisaje comprende colinas de sedimentos del Terciario de baja a media pendiente, con pastos como uso de la tierra dominante. Los suelos están formados a partir de sedimentos del Río Cauca 
y presentan una erosión de moderada a severa con manifestación de erosión, dejando expuesto el material parental (anfibolita, L. Arias 2011, com. pers.). Las plantaciones objeto de estudio contaban al momento del estudio, con cinco años de edad, siendo establecidas en el año 2004 sobre terrenos degradados por sobrepastoreo, en los que se ha perdido gran parte del horizonte Ap por erosión. Las plantaciones no recibieron ningún tipo de práctica silvícola, tenían una densidad promedio de rodal de 1 033 individuos/ha, un área basal de $1.4 \mathrm{~m}^{2} /$ ha y una altura de dominantes de $3.8 \mathrm{~m}$.

Diseño experimental: Dadas las condiciones topográficas homogéneas y la coetaneidad de la plantación, el diseño comprendió un muestreo sistemático en rejilla, estableciendo en cada intersección una parcela permanente de $250 \mathrm{~m}^{2}$, para un total de 20 parcelas. En cada una se ubicaron tres trampas circulares con tela de malla fina $\left(0.5 \mathrm{~m}^{2}\right.$ cada una) para recolectar hojarasca fina quincenalmente durante un año (noviembre de 2009 a noviembre de 2010). La hojarasca recolectada fue separada por fracciones después de cada recolección: hojas de $A$. indica $(\mathrm{HN})$, hojas de otras especies (HOe), material leñoso (ML) ramas con diámetro $<2 \mathrm{~cm}$ y pequeños restos de corteza, material reproductivo (MR), y otros restos (OR). El material se secó en horno a $65^{\circ} \mathrm{C}$ hasta obtener peso constante, después se combinaron y homogenizaron las muestras de $\mathrm{HN}$ para cada dos períodos quincenales (un mes) y se tomó una sub-muestra para análisis químico. La descomposición de la hojarasca foliar de A. indica se estudió instalando 27 litter-bags (20x20cm, $2 \mathrm{~mm}$ de poro) con tres gramos de hojas senescentes secas de $A$. indica sobre la superficie del terreno. Se retiraron tres litterbags a $\operatorname{los}$ 14, 28, 56, 84, 126, 168, 269, 297 y 365 días. El material recolectado se limpió, se secó a $65^{\circ} \mathrm{C}$ hasta obtener peso constante (materia seca residual, MSR). Las muestras de las tres litter-bags recolectadas en cada evento se combinaron y homogenizaron para análisis químico (León et al. 2011).
Para evaluar la reabsorción de nutrientes foliares se recolectaron mensualmente, durante un año, hojas verdes maduras, fotosintéticamente activas, que no exhibieran síntomas de enfermedades y/o herbivoría, y que no correspondieran a estados extremos de edad. Se seleccionaron 10 individuos representativos por parcela, en ocho de las estudiadas. El material foliar recolectado mensualmente (ca. 400 hojas), se combinó y una sub-muestra fue seleccionada de cada combinación, para análisis químico.

Análisis de muestras: Para los tejidos foliares se determinaron los cationes $(\mathrm{Ca}, \mathrm{Mg}$, $\mathrm{K}$ ), usando espectrofotometría de absorción atómica. El P se determinó por el método de azul de molibdato, el C por Walkley \& Black (1934) y el N mediante Kjeldahl. La Fibra Detergente Ácido (FDA) y la Lignina acorde a Goering \& van Soest (1970). La celulosa se obtuvo de la diferencia entre Fibra Detergente Ácido (FDA) y Lignina. Para los análisis de suelos se tomó una muestra por parcela, hasta una profundidad de 10cm (500-1 000g). De los sitios localizados por fuera de las plantaciones se tomaron 14 muestras de suelo superficial (sitios testigo). Estos sitios testigo fueron seleccionados en pastizales degradados por sobrepastoreo, aledaños a los sitios plantados, de características similares a las que tendrían los lugares evaluados si no se hubieran establecido las plantaciones. No se realizaron comparaciones con áreas de referencia (sitios conservados), dado que no se encontraron puntos representativos con estas características, las zonas cercanas son todas de lomerío, dedicadas al pastoreo y se encuentran bajo el influjo del proceso de degradación. Para las muestras de suelo se determinaron las bases intercambiables $(\mathrm{Ca}, \mathrm{Mg}, \mathrm{K})$ usando acetato de amonio $\left(\mathrm{CH}_{3} \mathrm{COO} \mathrm{NH}_{4}\right) 1 \mathrm{~N}$ neutro, y medición por absorción atómica. El $\mathrm{P}$ disponible se determinó usando el método Bray- Kurtz y el $\mathrm{N}$ total mediante Kjeldahl. Se determinó además: densidad aparente (DA), estabilidad de agregados (EA) según metodología de Yoder (1936); $\mathrm{NH}_{4}$, mediante extracción con $\mathrm{KCl}$ $2 \mathrm{M}$ y lectura con electrodo de ión selectivo 
(Keeney \& Nelson 1982), y liberación de $\mathrm{CO}_{2}$ mediante respirometría en laboratorio (Anderson \& Domsch 1978).

Cálculos: La Tasa de Retorno Potencial de Nutrientes (TRP) vía hojarasca foliar fue calculada como el producto de la concentración del nutriente por la materia seca de la hojarasca foliar. La reabsorción de nutrientes foliares se calculó como la diferencia entre las cantidades de nutrientes en las hojas verdes y en las hojas muertas (Hagen-Thorn et al. 2006). Este valor, dividido por la cantidad del elemento en las hojas maduras, permite obtener una aproximación de la cantidad retranslocada, que multiplicada por 100, la expresa en porcentaje (Del Arco et al. 1991).

$$
\mathrm{R}=\mathrm{Chm}-\frac{\mathrm{Ch}}{\mathrm{Chm}} * 100
$$

Donde, Chm es la concentración del nutriente en las hojas maduras, Ch es la concentración del nutriente en la hojarasca foliar. La cantidad reabsorbida de cada nutriente ( $\mathrm{g} / \mathrm{ha} \cdot \mathrm{a}$ ) o Flujo de Reabsorción (Fr), se calculó como el producto entre la diferencia absoluta de la concentración media de las hojas maduras y de la hojarasca foliar, y la producción de hojarasca foliar. La eficiencia en el uso de nutrientes se determinó a partir del Índice de Eficiencia de Vitousek (1982)-IEV-, este índice define la eficiencia con la que es utilizado un nutriente, como la cantidad de materia seca producida en el desfronde, por unidad de ese nutriente contenida en él, es decir, el inverso de la concentración. La pérdida de peso en las litter-bags, se ajustó a un modelo simple exponencial (Olson 1963):

$$
\frac{X_{t}}{X_{0}}=e^{-k t}
$$

Donde, $X_{t}$ es el peso del material remanente en el momento t, $X_{0}$ es el peso del material seco inicial, "e" es la base del logaritmo natural, $k$ es la tasa de descomposición, y $t$ el tiempo. El tiempo requerido para que se produzca la pérdida del $50 \%$ y $99 \%$ de la materia seca se calculó como $\mathrm{t}_{0.5}=-0.693 / k \mathrm{y} \mathrm{t}_{0.99}=-4.605 / k$, respectivamente. Se ajustaron modelos mediante regresión no lineal para la pérdida de peso del material foliar depositado en litter-bags. Se aplicó análisis de correlación lineal (r Pearson, $\mathrm{p}=0.05$ ) para determinar asociaciones entre las tasas de pérdida de peso y la calidad del sustrato y la precipitación. Para la selección de los modelos se emplearon el coeficiente de determinación $\left(\mathrm{R}^{2}\right)$, el estadístico Durbin-Watson (D-W), y la suma de cuadrados del error. Para la comparación de los valores de los parámetros de suelos entre los sitios testigo y las plantaciones, se empleó la prueba de comparación de medianas Mann- Whitney (Wilcoxon) para dos grupos $(\mathrm{p}=0.05)$.

\section{RESULTADOS}

Producción de hojarasca y retorno potencial de nutrientes: En la caída de hojarasca fina predominó la fracción foliar $(65 \%)$, siendo la participación similar para $\mathrm{HN}$ y $\mathrm{HOe}$ (Cuadro 1). Únicamente la fracción MR mostró asociación positiva y significativa con la precipitación ( $\mathrm{r}$ Pearson $=0.51, \mathrm{p}=0.05$ ). Las concentraciones de nutrientes en la hojarasca foliar y los retornos potenciales respectivos, estuvieron dominados por Ca y N, mientras que los menores valores se obtuvieron para $\mathrm{P}$ (Cuadro 2).

Descomposición de hojarasca y liberación de nutrientes: Después de un año la Materia Seca Residual (MSR) alcanzó en promedio $31.8 \%$ del material inicialmente depositado en las litter-bags (Fig. 1). La mayoría de los parámetros de calidad de dicho material se incrementaron al finalizar el estudio (Cuadro $3)$, sin embargo no se encontró una asociación significativa $(\mathrm{p}>0.05)$ a partir de los coeficientes de correlación de Pearson, entre la MSR y los parámetros de calidad de hojarasca, ni con la precipitación. A partir de la constante anual de descomposición $(k)$ del modelo simple exponencial negativo $(k=1.58)$, el tiempo necesario para que se descomponga el $50 \%$ del material $\left(\mathrm{t}_{0.5}\right)$ sería de 0.44 años (161 días) y de 


\section{CUADRO 1}

Caída de hojarasca fina $(\mathrm{kg} / \mathrm{ha} \pm \mathrm{DT})$ discriminada por fracciones, en las plantaciones de $A$. indica en Santa Fe de Antioquia, Colombia

TABLE 1

Fine litterfall production $(\mathrm{kg} / \mathrm{ha} \pm \mathrm{sdtv})$ by fraction in $A$. indica plantations in Santa Fe de Antioquia, Colombia

\begin{tabular}{lcccccc}
\multicolumn{1}{c}{ Período } & \multicolumn{5}{c}{ Fracciones } \\
& HN & HOe & MR & ML & OR & HT \\
Promedio mensual & $15.4 \pm 10.8$ & $14.7 \pm 6.1$ & $11.3 \pm 11.6$ & $4.0 \pm 1.7$ & $1.0 \pm 0.7$ & $46.5 \pm 21.7$ \\
Total anual & 184.8 & 176.9 & 135.8 & 48.1 & 11.9 & 557.5 \\
Participación relativa anual (\%) & 33 & 32 & 24 & 9 & 2 & 100 \\
\hline
\end{tabular}

HN: hojas de A. indica, HOe: hojas de otras especies, MR: material reproductivo, ML: material leñoso, OR: otros restos, HT: hojarasca total HN: A. indica leaves, HOe: leaves from other species, MR: reproductive material, ML: wood material, OR: other materials, HT: total fine litterfall detached from the canopy DT: desviación típica. sdtv: standard deviation.

\section{CUADRO 2}

Concentración de nutrientes en la hojarasca foliar de $A$. indica $(\% \pm \mathrm{DT})$ y retorno potencial respectivo $(\mathrm{kg} / \mathrm{ha} \pm \mathrm{DT})$ en plantaciones de $A$. indica en Santa Fe de Antioquia, Colombia

\section{TABLE 2}

Nutrient concentration $(\% \pm$ sdtv $)$ and potential return $(\mathrm{kg} / \mathrm{ha} \pm \mathrm{sdtv})$ values through foliar litterfall of $A$. indica plantations in Santa Fe de Antioquia, Colombia

\begin{tabular}{lccccc}
\multicolumn{1}{c}{ Parámetro } & $\mathrm{P}$ & $\mathrm{N}$ & $\mathrm{Ca}$ & $\mathrm{Mg}$ & $\mathrm{K}$ \\
Concentración media mensual & $0.03 \pm 0.006$ & $1.29 \pm 0.311$ & $2.16 \pm 0.649$ & $0.46 \pm 0.064$ & $0.29 \pm 0.144$ \\
& $(19.05)$ & $(24.18)$ & $(30.06)$ & $(13.88)$ & $(49.31)$ \\
Retorno medio mensual & $0.005 \pm 0.004$ & $0.203 \pm 0.180$ & $0.385 \pm 0.440$ & $0.074 \pm 0.061$ & $0.043 \pm 0.028$ \\
& $(86.67)$ & $(114.39)$ & $(82.04)$ & $(64.53)$ & $(89.03)$ \\
Retorno total anual & 0.06 & 2.43 & 4.62 & 0.89 & 0.52 \\
\hline
\end{tabular}

Entre paréntesis, coeficiente de variación (\%). DT: desviación típica.

The variation coefficient is noted in parenthesis (\%). sdtv: standard deviation.

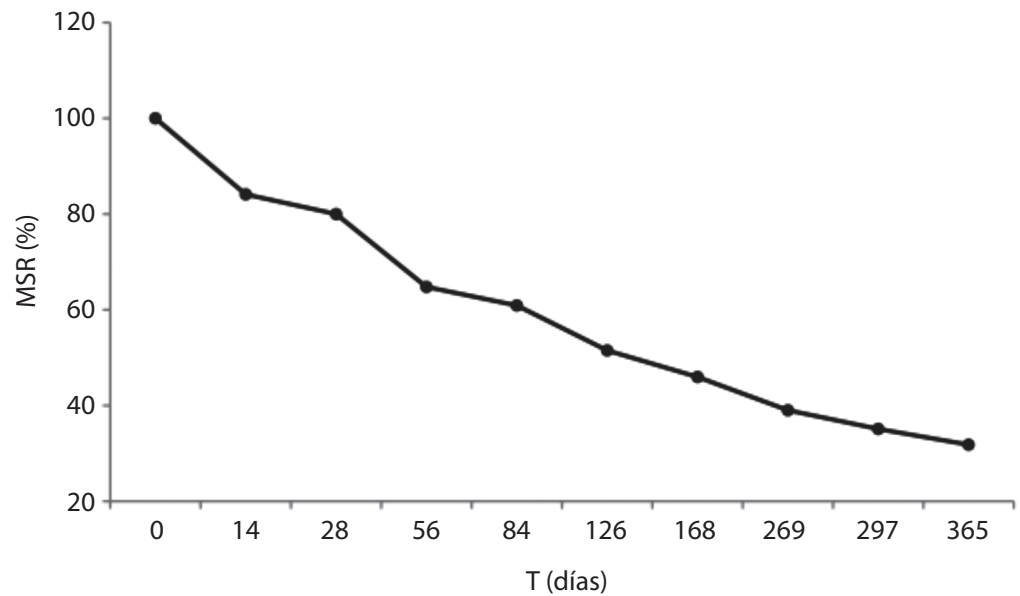

Fig. 1. Evolución temporal de la Materia Seca Residual (MSR) de la hojarasca foliar depositada en las litter-bags en plantaciones de $A$. indica en Santa Fe de Antioquia, Colombia. Cada punto representa el promedio de tres litter-bags.

Fig. 1. Temporary evolution of the residual dry matter (MSR) of the foliar litterfall deposited in the litter-bags in $A$. indica plantations in Santa Fe de Antioquia, Colombia. Each point represents the average of three litter-bags. 
CUADRO 3

Parámetros de calidad del material foliar depositado en las litter-bags en plantaciones de $A$. indica en Santa Fe de Antioquia, Colombia

TABLE 3

Quality parameters for the foliar material deposited in the litter-bags in A. indica plantations in Santa Fe de Antioquia, Colombia

\begin{tabular}{lcccccccc}
\multicolumn{1}{r}{ Valores } & $\mathrm{C}$ & $\mathrm{N}$ & $\mathrm{P}$ & $\mathrm{C} / \mathrm{N}$ & $\mathrm{N} / \mathrm{P}$ & Lignina (\%) & Lignina/N & Celulosa (\%) \\
Iniciales & & $(\%)$ & & & & & & \\
Finales & 27.26 & 0.70 & 0.03 & 38.94 & 23.33 & 10.40 & 14.86 & 23.50 \\
\hline
\end{tabular}

2.91 años (1 062 días) para una descomposición de $99 \%\left(\mathrm{t}_{0.99}\right)$.

Al finalizar el estudio se encontró el patrón decreciente $\mathrm{K}>\mathrm{P}>\mathrm{Mg}>\mathrm{Ca}>\mathrm{N}$ para las cantidades remanentes de nutrientes (Fig. 2). La máxima liberación se produjo para K $(99.9 \%)$ y la mínima para $\mathrm{N}(40.9 \%)$. Al comienzo del estudio la liberación de $\mathrm{N}$ fue lenta, predominando luego la inmovilización.

Reabsorción y eficiencia en el uso de nutrientes: Las mayores concentraciones de nutrientes en las hojas maduras fueron de $\mathrm{N}$ y $\mathrm{Ca}$, y las menores las de P (Cuadro 4). La mayor eficiencia en el uso de nutrientes se encontró para el $\mathrm{P}(\mathrm{IEV}=3$ 175.5) y la menor para el $\mathrm{Ca}$ $(\mathrm{IEV}=49.9)$. La mayor reabsorción de nutrientes foliares se observó para el $\mathrm{K}(\mathrm{R}=60 \%)$, mientras que el $\mathrm{Mg}$ con $\mathrm{R}=6.8 \%$, se comportó como el nutriente menos móvil. En términos absolutos, el mayor flujo de nutrientes reabsorbidos anualmente fue el de N (ca. 1390g/ha) y los menores los de Mg y P (ca. $30 \mathrm{~g} / \mathrm{ha})$.

Evolución de las propiedades edáficas: Al comparar los parámetros químicos edáficos de las plantaciones con los de los sitios Testigo, se observó un patrón de mejoramiento

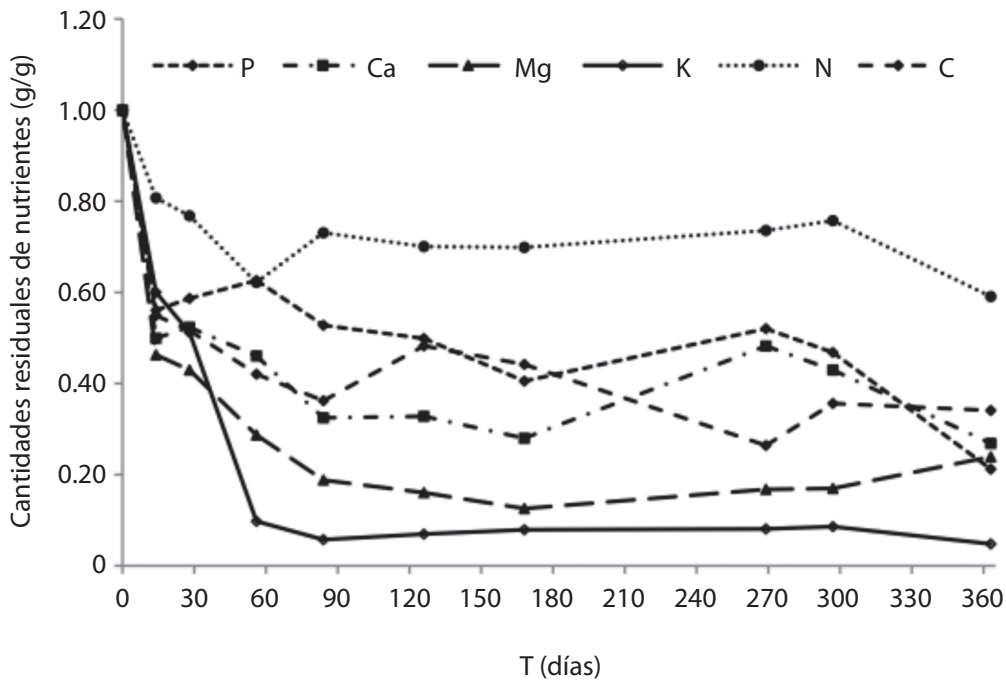

Fig. 2. Evolución temporal de las cantidades residuales de nutrientes $(\mathrm{g} / \mathrm{g})$ en las litter-bags en plantaciones de $A$. indica en Santa Fe de Antioquia, Colombia.

Fig. 2. Temporal evolution of the residual quantities of nutrients $(\mathrm{g} / \mathrm{g})$ in the litter-bags in A. indica plantations in Santa Fe de Antioquia, Colombia. 
CUADRO 4

Valores medios mensuales de concentración de nutrientes en hojas maduras (Chm), reabsorción foliar (R) y eficiencia en el uso de nutrientes (IEV) $\pm \mathrm{DT}$ en plantaciones de $A$. indica en Santa Fe de Antioquia, Colombia

TABLE 4

Mean monthly values for nutrient concentrations in mature leaves $(\mathrm{Chm})$, nutrient resorption $(\mathrm{R})$ and nutrient use efficiency (IEV) \pm sdtv in $A$. indica plantations in Santa Fe de Antioquia, Colombia

\begin{tabular}{ccccc} 
Elemento & Chm $(\%)$ & $\mathrm{R}(\%)$ & $\mathrm{IEV}$ & $\mathrm{Fr}(\mathrm{g} / \mathrm{ha} \cdot \mathrm{a})$ \\
$\mathrm{P}$ & $0.05 \pm 0.01$ & $35.0 \pm 11.2$ & $3175.5 \pm 737.1$ & 32.0 \\
$\mathrm{Ca}$ & $1.95 \pm 0.65$ & $\mathrm{nc}$ & $49.9 \pm 14.1$ & $\mathrm{nc}$ \\
$\mathrm{Mg}$ & $0.50 \pm 0.06$ & $6.8 \pm 14.5$ & $221.8 \pm 34.0$ & 32.2 \\
$\mathrm{~K}$ & $0.80 \pm 0.18$ & $60.0 \pm 26.2$ & $420.8 \pm 199.2$ & 928.4 \\
$\mathrm{~N}$ & $2.07 \pm 0.32$ & $38.2 \pm 11.0$ & $82.1 \pm 19.9$ & 1385.7 \\
\hline
\end{tabular}

Fr: Flujo anual de reabsorción foliar.

nc: no calculado.

Fr: Yearly foliar resorption flux. nc: not calculated.

DT: desviación típica.

sdtv: standard deviation.

(Cuadro 5). Tales mejoramientos se determinaron para MO, DA, N, P, K y actividad microbiológica, reflejada en liberación de $\mathrm{CO}_{2}$.

\section{DISCUSIÓN}

Producción de hojarasca y retorno potencial de nutrientes: Los aportes de hojarasca fina (Cuadro 1) fueron inferiores a los reportados para plantaciones forestales de tierras bajas tropicales (ca. 5-10Mg/ ha:a: Goma-Tchimbakala \& Bernhard-Reversat 2006, Barlow et al. 2007) y a los señalados por Singh et al. (1999) para plantaciones de Azadirachta indica en suelos degradados por minería (2 990kg/ha'a para hojarasca foliar y 3 $620 \mathrm{~kg} / \mathrm{ha}$ a para hojarasca total). Dada la falta de prácticas de manejo de las plantaciones, y la falta de cierre del dosel, se facilitó la presencia de especies pioneras, dando lugar a un alta participación de hojas de otras especies (HOe), principalmente Byrsonima sp., que han derivado ventajas para la presencia de los árboles de A. indica plantados.

Las concentraciones medias de nutrientes en la hojarasca foliar de $A$. indica siguieron la secuencia $\mathrm{Ca}>\mathrm{N}>\mathrm{Mg}>\mathrm{K}>\mathrm{P}$, siendo los valores de $\mathrm{Ca}$ superiores a los de bosques secos de tierras bajas tropicales $(1.7 \mathrm{~kg} / \mathrm{ha} \cdot \mathrm{a}:$ Singh et al.
2004). Estas altas concentraciones de $\mathrm{Ca}$, así como las de $\mathrm{Mg}$ ( $c a .0 .5 \%)$, coinciden con su disponibilidad edáfica, que favorece su captura y asimilación. Por el contrario, las concentraciones foliares de $\mathrm{K}$ ( $c a$. $0.3 \%$ ) se ubicaron en el extremo inferior del intervalo pantropical (0.27 \pm 0.11 : Duivenvoorden \& Lips 1995). Esta escasez de K podría ser el reflejo de las características edáficas, condicionadas por la alta participación del $\mathrm{Mg}$, que siendo superior en proporción a la del $\mathrm{Ca}$, genera una relación $\mathrm{Ca} / \mathrm{Mg}<1(0.96)$ y una mayor absorción de $\mathrm{Mg}$ (Marín 1986), limitando en consecuencia las concentraciones de $\mathrm{K}$.

Las concentraciones de $\mathrm{N}$ en la necromasa foliar fueron cercanas a las reportadas por Singh et al. $1999(1.0 \%)$ y por Jha \& Mohapatra 2010 (ca. 1.2\%) en plantaciones similares, representando una disponibilidad aceptable del elemento. El mayor déficit de nutrientes se encontró para $\mathrm{P}$, siendo alta la relación N/P (43), superando en casi cuatro órdenes de magnitud la sugerida como crítica (11.9: Aerts 1997). La mayor variabilidad temporal en la concentración de nutrientes la mostró el $\mathrm{K}$, resultado de su carácter móvil (Hagen-Thorn et al. 2006). Los retornos anuales de $\mathrm{N}$ y $\mathrm{P}$ vía hojarasca fina, fueron muy inferiores a los reportados para plantaciones 
CUADRO 5

Valores medios de parámetros edáficos en sitios dentro y fuera (testigos) \pm DT de las plantaciones de $A$. indica en Santa Fe de Antioquia, Colombia

TABLE 5

Mean values for soil parameters in sites within and outside (controls) \pm sdtv of the A. indica plantations in Santa Fe de Antioquia, Colombia

\begin{tabular}{lccc}
\multicolumn{1}{c}{ Parámetros } & Rodales $(\mathrm{n}=20)$ & Testigos $(\mathrm{n}=14)$ & ICP \\
$\mathrm{pH}$ & $6.38 \pm 0.45$ & $6.31 \pm 0.57$ & 1.01 \\
$\mathrm{MO}(\%)$ & $3.44 \pm 1.31^{\mathrm{b}}$ & $2.00 \pm 0.94^{\mathrm{a}}$ & 1.72 \\
$\mathrm{EA}(\%)$ & $80.07 \pm 9.51$ & $72.87 \pm 10.60$ & 1.10 \\
$\mathrm{DA}\left(\mathrm{g} / \mathrm{cm}^{3}\right)$ & $1.25 \pm 0.09^{\mathrm{a}}$ & $1.35 \pm 0.11^{\mathrm{b}}$ & 0.93 \\
$\mathrm{~N}(\%)$ & $0.27 \pm 0.04^{\mathrm{b}}$ & $0.21 \pm 0.09^{\mathrm{a}}$ & 1.27 \\
$\mathrm{P}(\mathrm{mg} / \mathrm{kg})$ & $4.32 \pm 1.30^{\mathrm{b}}$ & $3.30 \pm 0.88^{\mathrm{a}}$ & 1.31 \\
$\mathrm{Ca}(\mathrm{cmol}(+) / \mathrm{kg})$ & $7.10 \pm 3.58$ & $6.17 \pm 2.52$ & 1.15 \\
$\mathrm{Mg}(\mathrm{cmol}(+) / \mathrm{kg})$ & $7.35 \pm 2.95$ & $6.66 \pm 2.80$ & 1.10 \\
$\mathrm{~K}(\mathrm{cmol}(+) / \mathrm{kg})$ & $0.36 \pm 0.14^{\mathrm{b}}$ & $0.22 \pm 0.05^{\mathrm{a}}$ & 1.61 \\
$\mathrm{CICE}(\mathrm{cmol}(+) / \mathrm{kg})$ & $14.81 \pm 6.02$ & $13.05 \pm 4.59$ & 1.14 \\
$\mathrm{CO}(\mathrm{mg} / \mathrm{kg} \cdot \mathrm{día})$ & $11.70 \pm 0.3^{\mathrm{b}}$ & $10.00 \pm 1.7^{\mathrm{a}}$ & 1.17 \\
$\mathrm{NH}_{4}(\mathrm{mg} / \mathrm{kg})$ & $4.95 \pm 4.26$ & $4.08 \pm 2.26$ & 1.21 \\
\hline
\end{tabular}

DT: desviación típica. sdtv: standard deviation.

MO: materia orgánica, EA: estabilidad de agregados, DA: densidad aparente, CICE: capacidad de intercambio catiónico, $\mathrm{CO}_{2}$ : dióxido de carbono liberado por microorganismos. Letras a y b indican la existencia, o no, respectivamente, de diferencias significativas (Mann-Whitney, $\mathrm{p}=0.05$ ), ICP=Índice de Cambio por Parámetro (Valor parámetro en plantaciones/ Valor parámetro en sitios testigo).

MO: organic matter, EA: aggregate stability, DA: bulk density, CICE: cation exchange capacity, CO2: carbon dioxide released by microorganisms. $\mathrm{a}$ and $\mathrm{b}$ indicate the existence or non-existence, respectively, of significant differences (MannWhitney, $\mathrm{p}=0.05$ ), ICP=parameter change index (plantation parameter/control parameter).

de $A$. indica en la India $(29.9-66.4 \mathrm{~kg} / \mathrm{ha}$ a y 1.7-4.3kg/ha: : Singh et al. 1999, Jha \& Mohapatra 2010). Estas grandes diferencias se deben a la mayor producción de hojarasca en tales sitios (2990kg/haa y $5400 / \mathrm{kg} / \mathrm{ha} \cdot \mathrm{a})$, asociadas a mayores valores de densidad, biomasa y edad del rodal (Singh et al. 1999, Jha \& Mohapatra 2010). Por otra parte, los retornos potenciales de $\mathrm{N}$ y $\mathrm{P}$ vía hojarasca foliar del estudio se corresponden con los encontrados en bosques secos de la India y México (N: $2.0 \mathrm{~kg} / \mathrm{ha}$ a y P: 0.07-0.1 kg/ha'a: Singh et al. 2004, Cárdenas \& Campo 2007).

Descomposición de hojarasca y liberación de nutrientes: Al comienzo del estudio se presentó la mayor pérdida de peso del material en las litter-bags, y se observó hacia el día 56 una descomposición cercana al 35\%, luego fue más lenta. Altas pérdidas de peso en las primeras etapas del proceso, coinciden con lo reportado por Goma-Tchimbakala \& Bernhard-Reversat (2006) para Terminalia superba y bosque tropical natural, así como por Castellanos \& León (2011) en plantaciones de Acacia mangium. De acuerdo con Hernández \& Murcia (1992) y Fioretto et al. (2005), la calidad del sustrato determina su velocidad de descomposición, siendo reportados como buenos predictores, los contenidos de lignina, fenoles, $\mathrm{N}, \mathrm{C}$ y $\mathrm{P}$, y las relaciones $\mathrm{C} / \mathrm{N}, \mathrm{C} / \mathrm{P}$, y N/P (Martínez-Yrízar et al. 2007, Prause \& Fernández 2007, Castellanos \& León 2011). Aunque el contenido de lignina fue bajo en comparación con lo encontrado para otras plantaciones forestales subtropicales y tropicales 
(Kadir et al. 2001, Castellanos \& León 2011), ésta pareciera ejercer un control sobre la pérdida de peso. Al final del experimento, se encontraron incrementos de ella con respecto a los valores iniciales, que indica un aumento de formas recalcitrantes, y afecta la pérdida de peso del material remanente en las bolsas.

El parámetro N/P reflejó su efecto directo sobre la descomposición, conforme las mayores tasas de pérdida de peso del material foliar se asociaron con menores valores de éste, como resultado de la escasez de $\mathrm{P}$ en el suelo y en los órganos foliares, lo cual condiciona la actividad de los microorganismos descomponedores, disminuyendo la velocidad de descomposición. Según Aerts (1997), 11.9 es el valor crítico para la relación N/P en la hojarasca foliar. En los bosques tropicales, éste representa algún grado de escasez de P para los organismos descomponedores, ya que en las células de hongos y bacterias tal relación se encuentra alrededor de 10-15 (Swift et al. 1979). El valor N/P inicial en este estudio (23.3) evidencia las limitaciones de $\mathrm{P}, \mathrm{y}$ así su posible inmovilización biológica. La tasa de descomposición $(k=1.58)$, se localizó dentro del intervalo reportado para bosques y plantaciones de tierras bajas tropicales $(k=0.1-4.8$ : Sundarapandian \& Swamy 1999, Singh et al. 1999, Kurzatkowski et al. 2004). Esto tiene significativas repercusiones desde la perspectiva de recuperación de tierras degradadas, ya que dicho valor supone el aporte de importantes cantidades de compuestos orgánicos y nutrientes liberados desde la hojarasca, que contribuyen al restablecimiento de las propiedades edáficas y su actividad biológica. Singh et al. (1999) encontraron para plantaciones de Nim sobre suelos degradados por minería, una tasa de descomposición similar a la del presente estudio $(k=1.31)$, y consecuentemente una MSR semejante (27\%).

La tendencia a incrementarse las concentraciones de nutrientes en el material evaluado para descomposición, coincide con lo reportado por Singh et al. (2004). El patrón general de liberación de nutrientes estuvo dominado por el K, alcanzando al término del estudio su casi totalidad con respecto al contenido inicial, resultado de su carácter móvil (Parker 1983). Se verificó un patrón de liberación neta para los nutrientes restantes, con excepción del N, que experimentó una fase inicial de liberación, para luego dominar la inmovilización, conforme ha sido encontrado en otros estudios (Mtambanengwe \& Kirchmann 1995). Esto pudo producirse, por el aumento de la biomasa microbiana de organismos colonizadores (Cochran 1990, Schlesinger 1991) así como por la invasión del material por el micelio de los hongos (Castellanos \& León 2011), o por fijación atmosférica de $\mathrm{N}_{2}$ (Wood 1974). Estos factores pueden incrementar la concentración foliar de $\mathrm{N}$, con lo cual, a pesar de seguirse registrando pérdidas de la materia seca en las litter-bags hacia el final del estudio, las cantidades remanentes calculadas para este nutriente se incrementan o disminuyen poco. La baja mineralización del $\mathrm{N}$ podría explicarse también a partir de los parámetros de calidad del sustrato depositado en las litter-bags. Así, se ha argumentado que la mineralización del $\mathrm{N}$ y la descomposición de la materia orgánica son rápidas cuando la relación $\mathrm{C} / \mathrm{N}$ es inferior a 25-40 y el contenido de $\mathrm{N}$ supera el intervalo 1.0-2.5\% (Torreta \& Takeda 1999 y Ngoran et al. 2006), condiciones diferentes a las de este estudio (C/N: 38.94 y N: 0.7\%).

Las cantidades residuales de $\mathrm{P}$ tendieron a disminuir, coincidiendo con lo reportado por Ngoran et al. (2006), para Acacia auriculiformis y Acacia mangium, aunque en algunos momentos se observó su inmovilización. Dadas las bajas concentraciones de $\mathrm{P}$ en el material foliar, la liberación efectiva se produjo principalmente como resultado de las pérdidas de peso experimentadas por las hojas en las litter-bags, antes que por el efecto de la concentración del elemento. La liberación efectiva de Ca y Mg (ambos alcalinos), cuyo patrón de liberación fue muy similar, con aproximadamente $75 \%$ en ambos casos, se produce como resultado de la baja acidez del suelo y su disponibilidad en el complejo de cambio, por lo tanto no hay una limitación de la actividad microbiológica, conforme ocurre en los suelos 
ácidos, donde son inmovilizados biológicamente (Duchaufour 1984).

Concentración, reabsorción y eficiencia en el uso de nutrientes: Al igual que en la hojarasca foliar, en las hojas maduras las mayores concentraciones fueron de $\mathrm{N} \mathrm{y} \mathrm{Ca}$, dada su disponibilidad edáfica. La menor eficiencia en el uso de nutrientes (IEV) se obtuvo para $\mathrm{Ca}$, dada su mayor concentración en la hojarasca y una baja reabsorción, la cual pudo evidenciarse, conforme las concentraciones en las hojas maduras fue muy similar a la de las hojas muertas, consecuencia de la baja movilidad del Ca (Landsberg \& Gower 1997). La adecuada disponibilidad de $\mathrm{N}$ se reflejó en la eficiencia en su uso (IEV=ca. 82), superior a la reportada para plantaciones de Nim por otros estudios en zonas semiáridas $(\mathrm{IEV}=c a .40-44$ : Deans et al. 2003, Agyarko et al. 2006).

La menor movilidad y alta disponibilidad edáfica del $\mathrm{Mg}$, condujeron a un mínimo flujo de reabsorción (Fr). El valor de la relación $\mathrm{N}: \mathrm{Mg}$ (4.14) de las hojas maduras, inferior a 17.5 , indicó la inexistencia de defíciencias críticas de $\mathrm{Mg}$ con respecto a N (Zas \& Serrada 2003). Niveles altos de $\mathrm{Mg}$ en hojas maduras de Nim fueron reportados por Drechsel et al. 1991, superando hasta en un 54\% las de plantaciones de A. auriculiformis y Albizia lebbek. Las concentraciones de P en las hojas maduras fueron inferiores a las reportadas por Radwanski \& Wickens (1981), Uyovbisere \& Elemo (2002), Deans et al. (2003) y Agyarco et al. (2006) en Nim (0.12-0.50\%).La relación $\mathrm{P}: \mathrm{N}$ (0.02), reflejó la extrema escasez de $\mathrm{P}$ en el suelo, coincidiendo con lo reportado por Medina et al. (1990). La relación N:P (41.4) fue muy superior al valor medio (16) reportado por Aerts (1997) para la hojarasca foliar en la región tropical, a partir del cual se establece una deficiencia crítica de $\mathrm{P}$ con respecto a $\mathrm{N}$ en la hojarasca foliar, derivándose una alta reabsorción $(\mathrm{R}=35 \%)$ y un uso eficiente (IEV=3 175.5), similar al obtenido en bosques secos de Borneo (IEV=3 382-3 686: Moran et al. 2000), lo que presupone una economía estrecha en el uso del nutriente dada su baja disponibilidad
(León et al. 2009). A pesar de las limitaciones de $\mathrm{P}$ encontradas, su reabsorción fue inferior a la de otros bosques secos (65\%: Murphy \& Lugo 1986), pero coincidió con los valores señalados por Cavelier (1996) para bosques tropicales de tierras bajas (29-50\%), donde usualmente se presentan limitaciones de $\mathrm{P}$ para la nutrición vegetal. La fuerte limitación por $\mathrm{P}$ quedó reflejada al comparar la eficiencia de uso, con los reportes de concentraciones para hojarasca foliar de Nim en Ghana (IEV=ca.714: Agyarko et al. 2006), en zonas semiáridas de Senegal $(\mathrm{IEV}=c a$. 833: Deans et al. 2003), y en suelos degradados por minería en la India (IEV=ca. 2000: Singh et al. 1999), en donde los valores IEV fueron inferiores.

Aunque en menor medida que el $\mathrm{P}$, el $\mathrm{K}$ representó limitaciones para las plantaciones de Nim, debido al desbalance edáfico $\mathrm{Ca} / \mathrm{Mg}$ que incide a su vez en la disponibilidad del K (Marín 1986). El valor K:Ca de las hojas maduras (ca. 0.4) fue inferior al umbral crítico en la nutrición de $\mathrm{K}(\mathrm{K}: \mathrm{Ca}=0.5$ : Ballard \& Carter 1986). Asimismo, el valor $\mathrm{K}: \mathrm{N}<0.65 \mathrm{en}$ las hojas maduras (0.4), determinó deficiencias en la nutrición de $\mathrm{K}$ con respecto a $\mathrm{N}$. Como consecuencia de la limitación por $\mathrm{K}$, se produjo su máxima reabsorción, y su ubicación en un segundo lugar en eficiencia en su uso (IEV $=c a$. 420) y en las cantidades anuales reabsorbidas $(\mathrm{Fr}=c a .930 \mathrm{~g} / \mathrm{ha})$. Asimismo, la alta eficiencia en el uso de $\mathrm{K}$ indicó la limitación por el nutriente, superior a la reportada para un grupo de ocho especies plantadas en zonas semiáridas de Senegal (IEV=73-217: Deans et al. 2003), dentro de las cuales se incluyó Nim, registrando la eficiencia en el uso de K más alta, con un IEV=217, valor cercano a la mitad del índice obtenido para el presente estudio.

Evolución de las propiedades edáficas: Los resultados del presente estudio muestran que, a pesar de la corta edad de las plantaciones de Nim, su incidencia es positiva sobre las propiedades de los suelos. Así, los valores medios de parámetros como $\mathrm{DA}, \mathrm{MO}, \mathrm{CO}_{2}, \mathrm{~N}$, $\mathrm{P}$ y $\mathrm{K}$ mostraron un mejoramiento significativo entre los sitios testigo y los sitios plantados. 
La densidad aparente (DA) en las plantaciones disminuyó, como resultado de la descompactación del suelo por las raíces finas, dado que tras su muerte y posterior descomposición el espacio poroso se incrementa (Yüksek \& Yüksek 2011). Los aportes de materia orgánica vía hojarasca desprendida del dosel, se van acumulando y descomponiendo paulatinamente en las plantaciones, generando incrementos de la materia orgánica edáfica (MO) y contribuyendo por lo tanto a la disminución de la DA. Estos incrementos en materia orgánica (MO) resultaron principalmente de la producción de hojarasca foliar (HN y HOe), su acumulación como mantillo (HNM) y posterior descomposición (Lowry et al. 1988). Así, se reconoce que la hojarasca fina está dominada en su composición por la fracción foliar (Meentemeyer et al. 1982), y que representa una de las principales rutas de retorno de nutrientes en los ecosistemas terrestres, gracias a la actividad de los organismos que participan en la descomposición y mineralización de la hojarasca. De estos procesos se derivan aportes de $\mathrm{N}$ y $\mathrm{P}$ que son liberados a partir de restos orgánicos (Guariguata \& Kattan 2002). Otros estudios han reportado cantidades altas de MO para suelos plantados con Nim (Drechsel et al. 1991).

El significativo incremento de $\mathrm{N}$ en las plantaciones estudiadas con respecto a los sitios no plantados, fue asimismo reportado por Drechsel et al. (1991) para otras plantaciones de Nim de cinco años de edad en suelos degradados de Togo, donde se encontraron incrementos superiores al $21 \%$ en los contenidos de $\mathrm{N}$, con respecto a sitios con arbustos regenerados naturalmente, y hasta de $140 \%$ con respecto a pastizales abandonados. En Nigeria, plantaciones de Nim con 22 años de edad mostraron contenidos edáficos de $\mathrm{N}$ hasta 2.6 veces superiores a los de sitios abandonados, anteriormente usados con fines agrícolas (Radwanski 1969). Los incrementos de $\mathrm{P}$ edáfico en las plantaciones fue un $31 \%$ mayor con respecto a los sitios testigo, sin embargo, la concentración en ambos casos fue excesivamente baja, 4.32 y $3.30 \%$ para plantación y testigo, respectivamente, constituyendo un factor restrictivo para el desarrollo de los individuos. Incrementos de $\mathrm{P}$ de hasta un $32 \%$ para plantaciones de Nim respecto al testigo en Togo, fueron reportados por Drechsel et al. (1991).

El K registró los mayores incrementos con respecto a los sitios testigo, siendo altas sus concentraciones edáficas en las plantaciones, superando ampliamente lo reportado por Radwanski (1969) en plantaciones de Nim en zonas secas $(0.23 \mathrm{cmol}(+) / \mathrm{kg})$. La alta disponibilidad del $\mathrm{Mg}$ en el complejo de cambio superó ampliamente el límite sugerido por Guerrero $(1988)(\mathrm{Mg}>1 \mathrm{cmol}(+) / \mathrm{kg})$. El elevado contenido de $\mathrm{Mg}$ en el suelo se deriva del material parental (Anfibolita) como ocurre en la unidad geológica en que se desarrolló este estudio (AN/EVm (Pz-KT): Maya 2001 y González 2001). Aunque las concentraciones edáficas de $\mathrm{K}$ en las plantaciones fueron altas, su disponibilidad se vio afectada por la alta participación de $\mathrm{Mg}$ en el complejo de cambio (49.6\%), valor superior al reportado por Navarro (2003) como adecuado (8-15\%). Debido a la relación antagónica entre $\mathrm{Mg}$ y $\mathrm{K}$, se genera un desbalance $\mathrm{Mg} / \mathrm{K}$, cuyo valor en las plantaciones de Nim (20.4) fue superior al sugerido como balanceado por Stover \& Simmonds 1987 para otros cultivos $(\mathrm{Mg} / \mathrm{K}=3.3)$. La participación de $\mathrm{Ca}(47.9 \%)$ fue inferior a la reportada como óptima (70-75\%: Ibid.) y la de K (2.4\%) se correspondió con el nivel óptimo (2.0-2.5\%: Ibid.). Estas proporciones no cambiaron significativamente con respecto a las que existían previamente en los sitios testigo. La limitación por $\mathrm{K}$ pudo asimismo verse reforzada por la relación $\mathrm{Ca} / \mathrm{Mg}$, que en las plantaciones de Nim fue muy baja (0.96), lo cual supone una absorción anormalmente alta de $\mathrm{Mg}$ y en consecuencia una alteración en las concentraciones requeridas de $\mathrm{K}$.

Los incrementos de materia orgánica y nutrientes edáficos, asociados a los aportes de hojarasca, favorecieron el aumento en la actividad de los microorganismos del suelo. Se encontró un aumento en la estabilidad de agregados (EA) en las plantaciones de Nim, dada la mayor estructuración de los agregados edáficos por la acción de las raíces finas que 
ocupan el suelo superficial, y descomposición de la necromasa, resultado de los aportes de materia orgánica (Montagnini \& Sancho 1990). Bajo cobertura de plantaciones forestales, la acidez puede aumentar como consecuencia de la descomposición de detritos, debido a la liberación de ácidos orgánicos y a la producción de compuestos de naturaleza ácida por las raíces (Pal \& Sharma 2001). Para plantaciones de Nim han sido registrados aumentos significativos en el pH de suelos con esta especie en África, en este estudio no se observaron cambios en este parámetro.

Este modelo de restauración activa representado por plantaciones puras de Nim, mostró un potencial favorable para la recuperación de tierras secas degradadas por pastoreo, en las que el suelo superficial se ha perdido en su casi totalidad por erosión. Esto se produce aun a pesar de la ausencia de prácticas de manejo silvícola de las plantaciones. Así, bajo las actuales condiciones de degradación, los aportes continuos de hojarasca fina son muy valiosos para la recuperación de la actividad biológica del suelo y de su funcionamiento como sistema, con lo cual se revela el potencial de las plantaciones en actividades de recuperación de sitios degradados secos, mejorando propiedades edáficas en lapsos de tiempo relativamente cortos. El modelo de restauración activa vía plantaciones forestales con especies exóticas usualmente ha encontrado resistencia, sin embargo, se ha reconocido que de ellas se derivan muchos beneficios en términos del restablecimiento de la biodiversidad y de las funciones ecosistémicas.

Las condiciones de degradación severa de tierras demandan para su recuperación, el concurso inicial de especies que, como Nim, se adapten rápida y exitosamente, hagan un uso eficiente de los nutrientes limitantes, y restablezcan progresivamente el ciclo biogeoquímico. En este primer momento de intervención, el Nim estaría cumpliendo algunas de las funciones desarrolladas por las especies pioneras, de alta rusticidad y tolerancia a las condiciones de libre exposición. Los resultados de este estudio permiten determinar que el establecimiento de plantaciones de Nim, como modelo de restauración activa, representa una alternativa viable y efectiva, que favorece los procesos de recuperación de áreas degradadas, facilitando el acondicionamiento edáfico para el ingreso de nuevas especies y consecuente restauración del espacio tratado. Sin embargo, aunque la especie tiene la capacidad de establecerse y desarrollarse sin demandar estrictamente la fertilización y en general un manejo y cuidado de la plantación, es recomendable implementar actividades de este tipo si se quiere acelerar el proceso.

\section{AGRADECIMIENTOS}

A la Dirección de Investigación Universidad Nacional de Colombia por la financiación del proyecto "Rehabilitación de tierras en proceso de desertificación con plantaciones de Nim (Azadirachta indica) en el Occidente Antioqueño". Convocatoria Nacional de Investigación y de Creación artística de la Universidad Nacional de Colombia 2010-2012. Al Área de Biogeoquímica del Laboratorio de Ecología-Universidad Nacional de Colombia. CORANTIOQUIA. A.N. Marín, L.F. Osorio, J.C. Guingue, G.E. Mazo y N. Álvarez por el acompañamiento técnico.

\section{RESUMEN}

Azadirachta indica A. Juss (Nim) ha sido ampliamente empleada en procedimientos de restauración, por lo tanto se evaluó el potencial de sus plantaciones para restaurar tierras secas degradadas por sobrepastoreo, vía reactivación del ciclo biogeoquímico. En 20 parcelas de $250 \mathrm{~m}^{2}$, se instalaron trampas de hojarasca y litter-bags. Se tomaron muestras de hojas maduras y de suelos dentro y fuera de las plantaciones, y se determinaron sus contenidos elementales. Fueron monitoreados la caída de hojarasca, la descomposición de hojarasca y la reabsorción de nutrientes foliares durante un año. Los aportes anuales de hojarasca fina representaron $557.54 \mathrm{~kg} / \mathrm{ha}$ ( $33 \%$ hojas de Nim). Los mayores retornos potenciales de nutrientes vía foliar fueron de $\mathrm{Ca}(4.6 \mathrm{~kg} / \mathrm{ha})$ y N $(2.4 \mathrm{~kg} / \mathrm{ha})$ y los menores de $\mathrm{P}$ $(0.06 \mathrm{~kg} / \mathrm{ha})$. El $68 \%$ del material se descompuso tras un año. La mayor liberación de nutrientes fue de K (100\%) y la menor de $\mathrm{N}(40 \%)$. El P fue el nutriente más limitante, con baja disponibilidad edáfica y alta eficiencia en su uso según el Índice de Vitousek (IEV=3 176) y la reabsorción 
foliar (35\%). Estas plantaciones juveniles demostraron efectividad en la reactivación del ciclo biogeoquímico, que mejoraron parámetros edáficos, según incrementos de materia orgánica, P y K; 72\%, 31\% y 61\%, respectivamente. Además mejoraron la estabilidad de agregados y las tasas de respiración microbiana.

Palabras claves: rehabilitación de suelos, restauración ecológica, ciclo biogeoquímico, Nim (Azadirachta indica), producción de hojarasca, descomposición de hojarasca, retorno de nutrientes.

\section{REFERENCIAS}

Aerts, R. 1997. Climate, leaf litter chemistry and leaf litter decomposition in terrestrial ecosystems: a triangular relationship. Oikos 79: 439-449.

Agyarko, K., P.K. Kwakye, M. Bonsu \& O. Benjamin. 2006. Impact of application of Neem leaves and poultry manure on nutrient dynamics of a Haplic Acrisol. Arch. Agron. Soil Sci. 52: 687-695.

Anaya, G. 1986. Problemas de erosión y desertificación en América Latina. Suelos Ecuat.16: 7-22.

Anderson, J.P.E. \& K.H. Domsch. 1978. A physiological method for the quantitative measurement of microbial biomass in soils. Soil Biol. Bioch. 10: 215-221.

Ballard, T.M. \& R.E. Carter. 1986. Evaluating forest stand nutrient status. Land Management Report No. 20. Ministry of Forests, Victoria, Columbia Británica, Canadá.

Barlow, J., T.A. Gardner, L.V. Ferreira \& C.A. Peres. 2007. Litter fall and decomposition in primary, secondary and plantation forests in the Brazilian Amazon. For. Ecol. Manag. 247: 91-97.

Cárdenas, I. \& J. Campo. 2007. Foliar nitrogen and phosphorus resorption and decomposition in the nitrogenfixing tree Lysiloma microphyllum in primary and secondary seasonally tropical dry forests in Mexico. J. Trop. Ecol. 23: 107-113.

Castellanos, B. \& J.D. León. 2011. Descomposición de hojarasca y liberación de nutrientes en plantaciones de Acacia mangium (Mimosaceae) establecidas en suelos degradados de Colombia. Rev. Biol. Trop. 59: 113-128.

Cavelier, J. 1996. Enviromental factors and ecophysiological processes along altitudinal gradients in wet tropical mountains, p. 339-439. In S.S. Mulkey, R.L. Chazdon \& A.P. Smith (eds.). Tropical Forest Plant Ecophysiology. Chapman \& Hall, Nueva York, EEUU.

Celentano, D., R.A. Zahawi, B. Finegan, F. Casanoves, R. Osterta, R.J. Cole \& K.D. Holl. 2011. Restauración ecológica de bosques tropicales en Costa Rica: efecto de varios modelos en la producción, acumulación y descomposición de hojarasca. Rev. Biol. Trop. 59: 1323-1336.

Chakraborty, R.N. \& D. Chakraborty. 1989. Changes in soil properties under Acacia auriculiformis plantations in Tripura. Indian For. 115: 272-273.

Cochran, V.L. 1990. Decomposition of barley straw in subarctic soil in the field. Biol. Fertil. Soils 10: 227-232.

Deans, J.D., O. Diagne, J. Nizinski, D.K. Lindley, M. Seck, K. Ingleby \& R.C. Munro. 2003. Comparative growth, biomass production, nutrient use and soil amelioration by nitrogen-fixing tree species in semiarid Senegal. Forest Ecol. Manag. 173: 253-264.

Del Arco, J.M., A. Escudero \& G.M. Vega. 1991. Effects of site characteristics on nitrogen retranslocation from senescing leaves. Ecology 72: 701-708.

Drechsel, P., B. Glaser \& W. Zech. 1991. Effect of four multipurpose tree species on soil amelioration during tree fallow in Central Togo. Agroforest. Syst. 16: 193-202.

Duchaufour, P.H. 1984. Edafología. I. Edafogénesis y clasificación. Masson, Barcelona, España.

Duivenvoorden, J.M. \& J.F. Lips. 1995. A land-ecologycal study of soils, vegetation and plant diversity in Colombian Amazonia. Tropenbos Series 12. The Tropenbos Foundation, Wageningen, Amsterdam, Holanda.

Ferrari, A.E. \& I.G. Wall. 2004. Utilización de árboles fijadores de nitrógeno para la revegetación de suelos degradados. Rev. Fac. Agron. 105: 63-87.

Fioretto, A., C. Di Nardo, S. Papa \& A. Fuggi. 2005. Lignin and cellulose degradation and nitrogen dynamics during decomposition of three leaf litter species in a Mediterranean ecosystem. Soil Biol. Biochem. 37: 1083-1091.

Garten, C.T. 2002. Soil carbon storage beneath recently established tree plantations in Tennessee and South Carolina, USA. Biomass Bioener. 23: 93-102.

Goering, H.K. \& P.J. van Soest. 1970. Forage Fiber Analyses (apparatus, reagents, procedures, and some applications). ARS/USDA Handbook No. 379, Superintendent of Documents, US Government Printing Office, Washington, D.C., EEUU.

Goma-Tchimbakala, J. \& F. Bernhard-Reversat. 2006. Comparison of litter dynamics in three plantations of an indigenous timber-tree species (Terminalia superba) and a natural tropical forest in Mayombe, Congo. Forest Ecol. Manag. 229: 304-313.

González, H. 2001. Mapa Geológico del Departamento de Antioquia. Memoria Explicativa. INGEOMINAS, Bogotá, Colombia. 
Guariguata, M.R. \& G.H. Kattan. 2002. Ecología y conservación de bosques neotropicales. Cartago, Costa Rica. LUR, Cartago, Costa Rica.

Guerrero, R. 1988. El diagnóstico químico de la fertilidad del suelo, p. 151-210. In M. Silva (ed.). Fertilidad de Suelos, diagnóstico y control. Sociedad Colombiana de Ciencia del Suelo, Bogotá, Colombia.

Hagen-Thorn, A., I. Varnagiryte, B. Nihlgård \& K. Armolaitis. 2006. Autumn nutrient resorption and losses in four deciduous forest tree species. Forest Ecol. Manag. 228: 33-39.

Hernández, M.L. \& M.A. Murcia. 1992. Estimación de la productividad primaria de Espeletia grandiflora H \& B y Pinus patula Schl \& Cham en el páramo El Granizo, Cundinamarca, Colombia. Trabajo de grado, Universidad Nacional de Colombia, Santa Fé de Bogotá, Colombia.

Holl, K.D. 2002.Tropical moist forest restoration, p. 539558. In M.R. Perrow \& A.J. Davy (eds.). Handbook of ecological restoration. Cambridge, Cambridge, Inglaterrra.

Jha, P. \& K.P. Mohapatra. 2010. Leaf litterfall, fine root production and turnover in four major tree species of the semi-arid region of India. Plant Soil 326: 481-491.

Kadir, W.R., O.V. Cleemput \& A.R. Zaharah. 2001. Microbial respiration and nitrogen release patters of decomposing Acacia mangium leaf litter from Kemasul forest reserve, Malaysa. J. Trop. Forest Sci. 13: 1-12.

Keeney, D.R. \& D.W. Nelson. 1982. Nitrogen in organic forms, p. 643-698. In A.L. Page \& R.H. Miller (eds.). Methods of Soil Analysis. American Society of Agronomy, Madison, Wisconsin, EEUU.

Kurzatkowski, D., C. Martius, H. Höfer, M. Garcia, B. Förster, L. Beck \& P. Vlek. 2004. Litter decomposition, microbial biomass and activity of soil organisms in three agroforestry sites in central Amazonia. Nutr. Cycl. Agroecosys. 69: 257-267.

Landsberg, J.J. \& S.T. Gower. 1997. Applications of physiological ecology to forest management. Academic, Nueva York, EEUU.

Le Houèrou, H.N. 1975. Science, power and desertification. Meeting on desertization. University of Cambridge, Cambridge, Inglaterra.

Le Houèrou, H.N. 1976. Can desertization be halted? p. 1-15. In FAO (ed.). Conservation in arid and semiarid zones. FAO Conservation Guide No. 3, Roma Italia.

León, J.D., M.I. González \& J.F. Gallardo. 2009. Retranslocación y eficiencia en el uso de nutrientes en bosques del centro de Antioquia. Rev. Colombia Forestal 12: 119-140.

León, J.D., M.I. González \& J.F. Gallardo. 2011. Comparación del ciclo biogeoquímico en bosques naturales y plantaciones de coníferas en ecosistemas de alta montaña de Colombia. Rev. Biol. Trop. 59: 1883-1894.

Lowry, J.N., J.B. Lowry \& R. Jones. 1988. Enhanced grass growth below a canopy of Albizia lebbek. Nitrogen Fixing Tree Res. Rep. 6: 45-46.

Marín, G. 1986. Fertilidad de Suelos: con énfasis en Colombia. Manual de asistencia técnica No. 39. ICA, Bogotá, Colombia.

Martínez-Yrízar, A., S. Nuñez \& A. Búrquez. 2007. Leaf litter decomposition in a southern sonoran desert ecosystem, northwestern México: Effects of habitat and litter quality. Acta Oecologica 32: 291-300.

MAVDT (Ministerio de Ambiente, Vivienda y Desarrollo Territorial). 2004. Plan de Acción Nacional de Lucha Contra la Desertificación y la Sequía en Colombia. Ministerio de Ambiente, Vivienda y Desarrollo Territorial, Bogotá, Colombia.

Maya, M. 2001. Distribución, facies y edad de las rocas metamórficas en Colombia. INGEOMINAS, Bogotá, Colombia.

Medina, E., V. García \& E. Cuevas. 1990. Sclerophylly and oligotrophic environments: relationships between leaf structure, mineral nutrient content, and drought resistance in tropical rain forest of the upper Rio Negro region. Biotropica 22: 51-64.

Meentemeyer, V., E.O. Box \& R. Thompson. 1982. World patterns and amounts of terrestrial plant litter production. Bioscience 32: 125-128.

Mehrotra, V.S. 1998. Arbuscular mycorrhizal associations of plants colonizing coal mine spoil in India. J. Agr. Sci. 130: 125-133.

Montagnini, F. \& F. Sancho. 1990. Impacts of native trees on tropical soils: a study in the Atlantic lowlands of Costa Rica, Central America. Ambio 19: 386-390.

Moran, J., M.G. Barker, A.J. Moran, P. Becker \& S.M. Ross. 2000. A comparison of the soil water, nutrient status, and litterfall characteristics of tropical heath and mixed-dipterocarp forest sites in Brunei. Biotropica 32: 2-13.

Mtambanengwe, F. \& H. Kirchmann. 1995. Litter from a tropical savanna woodland (Miombo): chemical composition and $\mathrm{C}$ and $\mathrm{N}$ mineralization. Soil Biol. Biochem. 27: 1639-1651.

Murphy, P.G. \& A.E. Lugo. 1986. Ecology of tropical dry forest. Ann. Rev. Ecol. Syst. 17: 67-68.

Navarro, G. 2003. Química Agrícola. Mundi-Prensa, Madrid, España.

Ngoran, A., N. Zakra, K. Ballo, C. Kouamé, F. Zapata, G. Hofman \& O. Van Cleemput. 2006. Litter decomposition of Acacia auriculiformis Cunn. Ex Benth and Acacia mangium Willdunder coconut trees on quaternary sandy soils in Ivory Coast. Biol. Fert. Soils 43: 102-106. 
Olson, J.S. 1963. Energy storage and balance of producers and decomposer in ecological systems. Ecology 44: 322-331.

Pal, R.C. \& A. Sharma. 2001. Afforestation for reclaiming degraded village common land: a case study. Biomass Bioenerg. 21: 35-42.

Parker, G.G. 1983. Throughfall and stem flow in the forest nutrient cycle. Adv. Ecol. Res. 13: 57-133.

Prause, J. \& C. Fernández. 2007. Litter decomposition and lignin/cellulose and lignin/total nitrogen rates of leaves in four species of the Argentine Subtropical forest. Agrochimica 51: 294-300.

Radwanski, S.A. 1969. Improvement of Red Acid Sands by the Neem Tree (Azadirachta indica) in Sokoto, Northwestern State of Nigeria. J. Appl. Ecol. 6: 507-511.

Radwanski, S.A. \& G.E. Wickens. 1981. Vegetative fallows and potential value of the Neem tree (Azadirachta indica) in the tropics. Econ. Bot. 35: 398-414.

Reynolds, J.F. \& D.M. Stafford-Smith. 2002. Global Desertification: Do Humans Cause Deserts? Dahlem University, Berlin, Alemania.

Sayer, J. \& C. Elliot. 2005. The role of commercial plantations in forest landscape restoration, p. 379-383. In S. Mansourian, D. Vallauri \& N. Duddley (eds.). Forest restoration in landscapes. Beyond planting trees. Springer, Nueva York, EEUU.

Schlesinger, W.H. 1991. Biogeochemistry: an analysis of global change. Academic, New York, EEUU.

Schrautzer, J., A. Rinker, K. Jensen, F. Muller, P. Schwartze \& C. Dier-Ben. 2007. Succession and restoration of drained fens: perspectives from northwestern Europe, p. 90-120. In L.R. Walker, J. Walker \& R.J. Hobbs (eds.). Linking restoration and ecological succession. Springer, Nueva York, EEUU.

Singh, V. \& V.K. Garg. 2007. Phytoremediation of a sodic forest ecosystem: plant community response to restoration process. Not. Bot. Hort. Agrobot. ClujNapoca 35: 77-85.

Singh, K.P., P.K. Singh \& S.P. Tripathi. 1999. Litterfall, litter decomposition and nutrient release patterns in four native tree species raised on coal mine spoil at Singrauli, India. Biol. Fert. Soils 29: 371-378.

Singh, A., A.K. Jha \& S. Singh. 2000. Effect of nutrient enrichment on native tropical trees planted on singrauli coalfields, India. Restor. Ecol. 8: 80-86.

Singh, G., B. Singh, V. Kuppusamy \& N. Vala. 2002. Variations in foliage and soil nutrient composition in
Acacia tortilis plantations of different ages in NorthWestern Rajashtan. Indian Forest 128: 514-521.

Singh, R., R. Kumar \& M. Agrawal. 2004. Litter decomposition and nutrient release in relation to atmospheric deposition of $\mathrm{S}$ and $\mathrm{N}$ in a Dry Tropical Region. Pedobiologia 48: 305-311.

Stover, R. \& N. Simmonds. 1987. Bananas. Longman, Londres, Inglaterra.

Sundarapandian, S.M. \& P.S. Swamy. 1999. Litter production and leaf-litter decomposition of selected tree species in a tropical forest at Kodayar in the western Ghats, India. Forest Ecol. Manag. 123: 231-244.

Swift, M., O. Heal \& J. Anderson. 1979. Decomposition in terrestrial ecosystems. Studies in ecology. University of California, Berkeley, California, EEUU.

Torreta, N.K. \& H. Takeda. 1999. Carbon and nitrogen dynamics of decomposing leaf litter in a tropical hill evergreen forest. European J. Soil Biol. 35: 57-63.

UN (United Nations). 1994. UN Earth Summit. Convention on Desertification. UN Conference in Environment and Development, Rio de Janeiro, Brazil, June 3-14, 1992. DPI/SD/1576. United Nations, Nueva York, EEUU.

Uyovbisere, E.O. \& K.A. Elemo. 2002. Effect of tree foliage of locust bean (Parkia biglobosa) and neem (Azadirachta indica) on soil fertility and productivity of maize in a savanna alfisol. Nutr. Cycl. Agroecosys. 62: $115-122$.

Vitousek, P.M. 1982. Nutrient cycling and nutrient use efficiency. Am. Nat. 119: 553-572.

Walkley, A. \& I.A. Black. 1934. An examination of the Degtjareff method for determining organic carbon in soils: Effect of variations in digestion conditions and of inorganic soil constituents. Soil Sci. 63: 251-263.

Wood, T.G. 1974. Field investigation under decomposition of leaves of Eucalyptus delegatensis in relation to environmental factors. Pedobiologia 14: 343-371.

Yoder, R.E. 1936. A direct method of aggregate analysis of soils and a study of the physical nature of erosion losses. J. Am. Soc. Agron. 28: 337-351.

Yüksek, T. \& F. Yüksek. 2011. The effects of restoration on soil properties in degraded land in the semi-arid region of Turkey. Catena 84: 47-53.

Zas, R. \& R. Serrada. 2003. Foliar nutrient status and nutritional relationships of young Pinus radiate D. Don plantations in northwest Spain. Forest Ecol. Manag. 174: 167-176. 
